\title{
Epidemiología de malaria urbana en Quibdó, Chocó
}

\author{
Johanna Ochoa, Lyda Osorio \\ Centro Internacional de Entrenamiento en Investigaciones Médicas, Cali, Colombia.
}

Introducción. La malaria urbana es un problema de salud pública en Colombia y poco se conoce acerca de sus características epidemiológicas, las cuales es necesario conocer para la implementación de medidas de control.

Objetivo. Determinar las características epidemiológicas de la malaria en el área urbana del municipio de Quibdó, Chocó.

Materiales y métodos. Los casos de malaria diagnosticados en la ciudad de Quibdó entre marzo y julio del 2001 fueron clasificados como autóctonos e importados a partir de historias de viaje a áreas endémicas o lugar de residencia. Los casos autóctonos fueron ubicados en el mapa con el fin de identificar las zonas de riesgo a partir del cálculo de morbilidad estandarizada por edad y sexo.

Resultados. Durante el periodo de estudio se incluyeron 839 casos de malaria: $77 \%$ por Plasmodium falciparum; $19 \%$ por Plasmodium vivax y $4 \%$ fueron infecciones por ambas especies. El $24,4 \%$ de los casos por $P$. falciparum y $39,1 \%$ de los casos de $P$. vivax se clasificó como autóctono. La zona de mayor riesgo de malaria se ubicó en los barrios del suroriente de la ciudad.

Conclusiones. La transmisión de malaria es focalizada y las áreas de mayor riesgo de infección están ubicadas cerca de zonas con vegetación. La información obtenida en este estudio permite concentrar esfuerzos en las zonas de mayor riesgo y optimizar la utilización de recursos.

Palabras clave: malaria/epidemiología, Plasmodium falciparum, Plasmodium vivax, asentamientos urbanos, Colombia.

\section{Epidemiology of urban malaria in Quibdo, Choco.}

Introduction. Although urban malaria is a public health problem in Colombia, little is known about its epidemiological characteristics, needed for the implementation of rational control strategies.

Objective. To determine the epidemiological characteristics of malaria in the urban area of the municipality of Quibdó, in northwest Colombia.

Materials and methods. Malaria cases diagnosed in the city of Quibdó between March and July, 2001 were classified as autochthonous or imported based on travel histories to endemic areas and place of residency. Autochthonous cases were mapped and risk areas identified using sex and age standardized morbidity ratios.

Results. During the study period 839 malaria cases were included: $77 \%$ due to Plasmodium falciparum; $19 \%$ due to $P$. vivax and $4 \%$ infections due to both species. $24.4 \%$ of $P$. falciparum cases and $39.1 \%$ of $P$. vivax cases were classified as autochthonous. Those neighborhoods located in the southeast of the town showed the highest risk for malaria.

Conclusion. As in other urban areas, malaria transmission in the city of Quibdo is focal and the areas with the highest risk are located near vegetation zones. These results are useful to prioritize areas for intervention and the allocation of resources.

Keywords: malaria/epidemiology, Plasmodium falciparum, Plasmodium vivax, urban settlements, Colombia. 
Durante el año 2004 se registraron en Colombia 123.177 casos de malaria y 25 muertes (1), lo que convierte a esta enfermedad en una prioridad del control nacional. El departamento de Chocó está ubicado al noroccidente del país, a lo largo de la costa Pacífica, y es una de las áreas con mayor riesgo para malaria en Colombia. En el 2004, Chocó reportó 7.103 casos de malaria y siete muertes (1); la transmisión de malaria se presenta en todos los municipios del departamento y el riesgo varía de mediano ( $>1$ caso/1.000 habitantes) a alto ( $>10$ casos/ 1.000 habitantes) (2). Así mismo, existe transmisión local de malaria en ocho cabeceras urbanas del departamento, incluyendo a la capital Quibdó.

Estudios previos sugieren que Anopheles nuñeztovari y Anopheles darlingison los vectores involucrados en la transmisión de malaria en el área urbana de Quibdó, aunque la densidad de las formas adultas capturadas fue baja. Con respecto a criaderos de mosquitos, este mismo estudio identificó 66 criaderos potenciales de Anopheles sp, en seis (9\%) de los cuales se encontraron formas inmaduras (3). Los estanques para cría de peces (en uso y abandonados), excavaciones de minería, lagunas y charcos de agua lluvia fueron positivos para formas inmaduras de Anopheles nuñeztovari (3).

A pesar de que existe transmisión local, se ha detectado que los residentes del área urbana de Quibdó que se movilizan temporalmente hacia áreas rurales y otras áreas urbanas donde hay transmisión de malaria tienen un mayor riesgo de adquirir la infección comparados con quienes no viajan (4). Viajar a un área endémica hasta un mes y tres meses antes del inicio de los síntomas es un factor de riesgo importante para adquirir una infección por $P$. falciparum $(\mathrm{OR}=28,96)$ y $P$. vivax $(\mathrm{OR}=14,24)$. Así, las infecciones por malaria en Quibdó pueden ser adquiridas en la misma ciudad (casos autóctonos) o en el área rural, en otros municipios del departamento o en

\footnotetext{
Correspondencia:

Lyda Osorio, Avenida $1 \mathrm{~N}$ \# 3-03 Cali, Colombia.

Teléfono: + 572-6682164, fax: +572-6672989

lydaosorio@cideim.org.co
}

Recibido: 04/04/06; aceptado: 19/05/06 otros departamentos endémicos para malaria (casos importados). Sin embargo, la discriminación del origen de los casos no se realiza rutinariamente, lo cual impide conocer la magnitud del problema de malaria en el área urbana de Quibdó e identificar las zonas de la ciudad donde existe transmisión autóctona.

Para determinar las características epidemiológicas de la transmisión de malaria en el área urbana de Quibdó, el presente estudio utiliza la definición de caso autóctono e importado para discriminar el origen de las infecciones por $P$. falciparum y $P$. vivax en Quibdó con base en el sitio probable de origen de la infección y antecedentes de visita a áreas endémicas como se ha propuesto anteriormente (4). La información obtenida en este estudio brinda herramientas a las instituciones de salud para la formulación de una estrategia racional de control de la malaria en el área urbana de Quibdó, concentrando los esfuerzos en las zonas de mayor riesgo y optimizando la movilización de recursos.

\section{Materiales y métodos}

\section{Área de estudio}

Quibdó, la capital del departamento del Chocó, presenta un clima cálido de bosque húmedo tropical con una temperatura superior a los $28^{\circ} \mathrm{C}$, humedad relativa del $90 \%$ y precipitación anual de 6.000 a $8.000 \mathrm{~mm}$ (5). Se ubica a orillas del río Atrato y funciona como puerto comercial, sujeto a la continua movilización de personas desde y hacia el área rural y otros municipios (6). Para el año 2000, Quibdó tenía una población de 144.494 habitantes, de los cuales el 62\% (89.586 habitantes) vivía en la cabecera municipal (7). La población está constituida principalmente por afrocolombianos con diferentes grados de mestizaje, indígenas y blancos-mestizos (6). La población rural del municipio de Quibdó se dedica a la agricultura, la minería, la extracción de madera y la pesca (6). En el área urbana, la mayoría de las personas son empleados del gobierno y del sector educativo y el resto de la población está constituida por estudiantes, amas de casa y comerciantes que actúan como intermediarios en la extracción de madera y oro y en la pesca (6). El $82 \%$ de los habitantes de Quibdó tiene por lo 
menos una necesidad básica insatisfecha (NBI) (vivienda inadecuada, carencia de servicios básicos, hacinamiento crítico, inasistencia escolar y alta dependencia económica) (7).

En Quibdó, $P$. falciparum predomina con una proporción de $66 \%$, mientras que cerca de $33 \%$ de los casos se deben a $P$. vivax y el restante $1 \%$ se clasifica como infección mixta $(P$. falciparum y $P$. vivax) (8). Las actividades de control se concentran en el diagnóstico y tratamiento temprano de casos sintomáticos y actividades esporádicas de control vectorial. Para el año 2001, el servicio de diagnóstico se ofrecía en dos hospitales, un puesto de salud, cinco clínicas privadas, cuatro laboratorios clínicos y un microscopista privado.

\section{Diseño del estudio y procedimientos}

Se condujo un estudio descriptivo transversal en el cual se incluyeron todas las instituciones de salud y laboratorios clínicos públicos y privados que realizaban diagnóstico de malaria en la ciudad de Quibdó. Se propuso que todos los pacientes que fueran diagnosticados con gota gruesa positiva entre el 27 de marzo y 27 de julio de 2001, sin importar la especie de parásito, fuesen entrevistados usando un cuestionario estándar. El cuestionario solicitaba información sociodemográfica del paciente, uso de medidas de protección contra mosquitos, episodios previos de malaria, fecha de inicio de síntomas (fiebre), el lugar más probable de infección, lugar de residencia y, si era residente, se solicitaban las historias de viaje en el lapso de una, dos y tres semanas, uno, tres y seis meses y un año antes del inicio de los síntomas. A cada paciente se le asignó un código y la información se organizó en Epi Info 6.04d (WHO/CDC, 2001). El estudio fue aprobado por el Comité de Ética del London School of Hygiene and Tropical Medicine y del Centro de Internacional de Entrenamiento e Investigaciones Médicas.

\section{Definición de casos autóctonos e importados}

Los casos por $P$. vivax y $P$. falciparum fueron clasificados de acuerdo con el sitio probable de infección como autóctonos o importados. Todos los casos en residentes fuera del área urbana de Quibdó fueron clasificados como importados. Los casos por $P$. falciparum en residentes de la ciudad de Quibdó fueron clasificados como importados si éstos habían viajado a un área endémica hasta un mes antes del inicio de los síntomas. Respecto a las infecciones por $P$. vivax, se clasificaron como importados los casos de residentes de la ciudad de Quibdó que hubiesen viajado a un área endémica entre una semana y tres meses antes del inicio de los síntomas (4).

\section{Análisis de los datos}

Se determinaron las características sociodemográficas y las frecuencias relativas de los casos clasificados como autóctonos para infecciones por $P$. falciparum y $P$. vivax. Usando el programa Arcview GIS versión 3.1 (Environmental Systems Research Institute Inc, 1998) se ubicó el sitio de residencia de los casos autóctonos en un mapa digital de Quibdó con una escala de 1:2500 que se obtuvo del Instituto

Cuadro 1. Agrupación de barrios de la ciudad de Quibdó por zonas.

\begin{tabular}{|c|c|}
\hline Zonas & Barrios \\
\hline A & $\begin{array}{l}\text { Cristo Rey/César Conto/El silencio } \\
\text { Roma/AlamedaReyes/YescaGrande/Yesquita } \\
\text { Calle de las Águilas/Chambacú }\end{array}$ \\
\hline B & San Vicente/Valencia \\
\hline C & Kennedy/San José/Miraflores/La Paz \\
\hline D & Huapango/Tomas Pérez \\
\hline $\mathbf{E}$ & Monserrate/Alamos \\
\hline $\mathbf{F}$ & Reposo 1/ Reposo 2 \\
\hline G & Obrero \\
\hline H & Villa España \\
\hline I & Victoria 1/Samper/La Gloria \\
\hline $\mathbf{J}$ & Buenos Aires/Subestación/Mis Esfuerzos \\
\hline K & El Caraño \\
\hline $\mathbf{L}$ & Paraíso \\
\hline M & Porvenir/Santo Domingo \\
\hline $\mathbf{N}$ & $\begin{array}{l}\text { Julio Figueroa/San Judas/La Esmeralda } \\
\text { Cohimbra/Las Américas/Los Angeles }\end{array}$ \\
\hline 0 & $\begin{array}{l}\text { Las Margaritas/Los Laureles/El Minuto de Dios/ } \\
\text { El Bosque }\end{array}$ \\
\hline $\mathbf{P}$ & $\begin{array}{l}\text { Loma Las Brisas/Chamblún/San Martín } \\
\text { La Aurora/Viento Libre/Medrano } \\
\text { Las Mercedes/El Piñal }\end{array}$ \\
\hline $\mathbf{Q}$ & Niño Jesús/Pablo VI /Palenque/Playita \\
\hline $\mathbf{R}$ & Horizonte \\
\hline S & Las Palmas/Simón Bolívar/Alfonso López \\
\hline $\mathbf{T}$ & $\begin{array}{l}\text { Cabí/ Los Rosales/Victoria 2/Poblado } \\
\text { La Cascorba/Flores de Buenaños, Jardín, } \\
\text { Zona Minera }\end{array}$ \\
\hline U & Obapo \\
\hline
\end{tabular}


Geográfico de Colombia Agustín Codazzi. La ubicación espacial de los casos autóctonos se utilizó para definir las áreas de riesgo a nivel de zonas en el área urbana de Quibdó (cuadro 1). Las zonas consistían en agrupaciones de barrios limitados geográficamente, de tal manera que las zonas se diferenciaran claramente una de otra. La identificación de las áreas de riesgo se realizó con el total de casos autóctonos por especies y zona a partir de la tasa de morbilidad estandarizada (TME) por edad y sexo, usando el método de estandarización indirecta (9). No fue posible calcular la TME para la zona U (Barrio Obapo) porque no se tenía información del número de habitantes. Los datos de población se obtuvieron del censo llevado a cabo por el programa de control de malaria de DASALUD Chocó en el 2002.

\section{Resultados}

Durante el estudio se reportaron 1.053 casos de malaria, de los cuales 839 (79,7\%) fueron entrevistados. La mayoría de los casos entrevistados, $77 \%$ (647), fueron infecciones por $P$. falciparum; el $19 \%$ (156) por $P$. vivax y el restante $4 \%$ (33) fueron infecciones mixtas ( $P$. vivax y $P$. falciparum). Para el análisis, los casos de infecciones mixtas fueron incluidos tanto en el grupo de $P$. falciparum como en el de $P$. vivax. Un paciente en el grupo de $P$. falciparum que no dio información acerca de historia de viaje no se pudo clasificar y no se incluyó en el análisis. En total se analizaron 679 casos de $P$. falciparum y 189 de $P$. vivax.

De los 679 casos de $P$. falciparum, 300 (44,2\%) se diagnosticaron en habitantes del área urbana y $379(55,8 \%)$ en residentes de fuera de la ciudad. Todos los 379 casos diagnosticados en residentes de fuera de la ciudad se clasificaron como importados, mientras que del total de 300 casos en residentes de Quibdó, 166 (24,4\%) fueron clasificados como autóctonos y $134(19,7 \%)$ como importados.

De los 189 casos por $P$. vivax entrevistados, 110 $(58,2 \%)$ se diagnosticaron en residentes del área urbana y $79(41,8 \%)$ en residentes de fuera de Quibdó. De los 110 casos en residentes de la ciudad de Quibdó, 74 (39,1\%) fueron clasificados como autóctonos y 36 (19\%) como importados.
La incidencia de malaria autóctona durante el periodo de estudio fue ligeramente mayor en los hombres ( 2,85 casos $/ 1.000$ hombres) que en las mujeres (2,23 casos/ 1.000 mujeres). Con relación a la edad, la incidencia fue similar para los grupos de 0 a $4(2,74 / 1000), 5$ a $14(2,82 / 1000)$ y 15 a 44 años (2,62/1000), pero menor para los individuos de 45 años o más (1,43/1000). Las características sociodemográficas para los casos autóctonos de $P$. falciparum y $P$. vivax se muestran en el cuadro 2.

Al ubicar en el mapa los casos autóctonos, se encontró que las zonas $\mathrm{T}$ (al sur de la ciudad), $\mathrm{H}$ e I (al norte) y $F$ (al occidente) mostraron la incidencia más alta de infecciones por $P$. falciparum durante el periodo de estudio $(\geq 3$ casos/1.000 habitantes). De estas, las zonas T y

Cuadro 2. Características sociodemográficas y de malaria de los casos autóctonos por $P$. falciparum y $P$. vivax en residentes del área urbana de Quibdó.

\begin{tabular}{|c|c|c|}
\hline Características & $\begin{array}{c}\text { Casos } \\
\text { autóctonos } \\
P . \text { falciparum } \\
\mathrm{n}=166(69,2 \%)\end{array}$ & $\begin{array}{c}\text { Casos } \\
\text { autóctonos } \\
P . \text { vivax } \\
\mathrm{n}=74(30,8 \%)\end{array}$ \\
\hline & Edad (años) & 20 \\
\hline$<5$ & $21(12,65)$ & $8(11)$ \\
\hline $5-14$ & $51(30,72)$ & $20(27,02)$ \\
\hline $15-44$ & $82(49,4)$ & $38(51,3)$ \\
\hline$\geq 45$ & $12(7,23)$ & $8(11)$ \\
\hline \multicolumn{3}{|l|}{ Sexo } \\
\hline Mujer & $83(50)$ & $33(44,6)$ \\
\hline Hombre & $83(50)$ & $41(55,4)$ \\
\hline \multicolumn{3}{|l|}{ Raza } \\
\hline Negra & $139(83,7)$ & $46(62,2)$ \\
\hline Otra & $27(16,3)$ & $28(37,8$ \\
\hline \multicolumn{3}{|l|}{ Ocupación } \\
\hline Menor/estudiante & $92(55,4)$ & $41(56,9)$ \\
\hline Ama de casa & $31(18,7)$ & $10(13,9)$ \\
\hline Trabajador rural & $6(3,6)$ & $3(4,2)$ \\
\hline Otro & $37(22,3)$ & $18(25)$ \\
\hline \multicolumn{3}{|l|}{ Uso de antimaláricos } \\
\hline $\begin{array}{l}\text { Malaria en el año } \\
\text { anterior }\end{array}$ & $26 / 164(15,8)$ & $25 / 74(33,8)$ \\
\hline $\begin{array}{l}\text { Caso en el hogar en el } \\
\text { mes anterior }\end{array}$ & $55 / 163(33,7)$ & $27 / 74(36,5)$ \\
\hline $\begin{array}{l}\text { Uso de protección } \\
\text { contra mosquitos }\end{array}$ & $104(62,65)$ & $53 / 74(71,6)$ \\
\hline
\end{tabular}


$\mathrm{H}$ fueron las áreas con mayor riesgo según la TME (figura 1A). Para los casos autóctonos por $P$. vivax, la zona $T$ tuvo la incidencia más alta ( $\geq 18$ casos/1.000 habitantes/periodo de estudio), seguida de la zona $Q$ (al suroeste) y la zona $E$ (al noroeste) con $\geq 2$ casos $/ 1.000$ habitantes/periodo de estudio. El análisis de TME confirmó que estas tres zonas tuvieron el mayor riesgo de infecciones por P. vivax (figura 1B).

\section{Discusión}

La transmisión de malaria en el área urbana de Quibdó, según se concluye del análisis de casos autóctonos, es altamente focalizada tanto para infecciones por $P$. falciparum como para $P$. vivax, y se caracteriza por ser periurbana. En Quibdó, como en áreas urbanas de África, diversos factores podrían contribuir a la presencia de focos de malaria en zonas periurbanas, tales como la urbanización poco planeada, la deficiencia en la infraestructura de vivienda (10), la carencia de servicios públicos, el bajo nivel socioeconómico de la población que habita en los límites de la ciudad (11) y la cercanía de las casas a campos irrigados y en general a cuerpos de agua y territorios ricos en vegetación en donde posiblemente la disponibilidad de criaderos es mayor $(12,13)$. En contraste, las zonas altamente urbanizadas del centro de la ciudad con casas de concreto y sin vegetación presentan las incidencias más bajas y son negativas para criaderos (Freddy Córdoba, Programa de Control de Malaria Quibdó; comunicación personal) (11).

De todos estos factores, la distancia de las viviendas a los criaderos se constituye en uno de los principales determinantes del riesgo de malaria en las áreas urbanas (12,14-16), como lo ilustra un estudio en un área periurbana de Maputo, Mozambique, donde el riesgo de infección fue 6,2 veces más alto para las personas que vivían a menos de 200 metros de los criaderos comparado con los individuos que vivían a 500 metros o más (14). En nuestro caso no se tuvo información de la localización exacta de los criaderos para hacer este tipo de análisis.

El comportamiento periurbano de la malaria en el área urbana de Quibdó es similar al que se presenta en otras ciudades de Colombia como Buenaventura y Villavicencio. En Buenaventura,

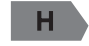

TME por edad y sexo

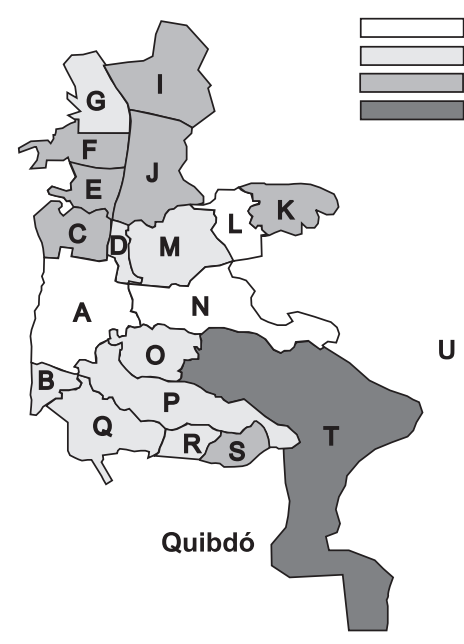

H)

TME por edad y sexo

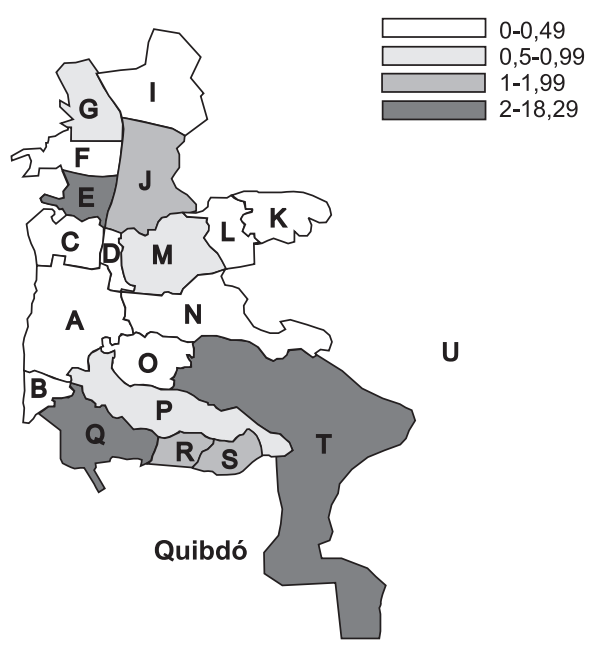

Colombia

Figura 1A. Tasa de morbilidad estandarizada por edad y sexo para las infecciones de P. falciparum en Quibdó, Chocó, marzo a julio de 2001. A - U: grupos de barrios.

Figura 1B. Tasa de morbilidad estandarizada por edad y sexo para las infecciones de P. vivax en Quibdó, Chocó, marzo a julio de 2001. A - U: grupos de barrios. 
los barrios que tradicionalmente presentan mayor número de casos están ubicados en el área periurbana (comunas 12, 10 y 9). Estas comunas están ocupadas por población que carece de servicios básicos, y la infraestructura de vivienda y los servicios de salud son deficientes (17). La comuna 12 reporta el mayor número de casos de malaria en Buenaventura y es allí donde se han encontrado la mayoría de criaderos positivos para Anopheles sp (17). En Villavicencio, la comuna 5 es una zona periurbana de invasión que ha tenido problemas de malaria desde hace 10 años por su proximidad al territorio selvático (Luz Estela Buitrago, Red de Entomología, SSDM, comunicación personal). Las tres ciudades son también similares en cuanto al tipo de criaderos urbanos, producto de actividades económicas de tipo rural. De esta manera, los estanques de cría de peces constituyen uno de los principales criaderos en las tres ciudades $(3,18,19)$, mientras que las excavaciones de minería son criaderos importantes en Quibdó y Buenaventura $(3,18)$.

En un estudio previo realizado en la ciudad de Quibdó se reportó la existencia de criaderos para Anopheles en excavaciones de minería de la Zona Minera, uno de los barrios que conforman la zona Ty que en este estudio presentó el mayor riesgo para infecciones por $P$. falciparum y $P$. vivax (3). Estos hallazgos señalan la necesidad de realizar vigilancia en las excavaciones de minería en uso y abandonadas que acumulen agua en esta área. Además de la zona T, en las zonas E, F, H, I, S y $Q$, deben ser prioritarios los estudios entomológicos que permitan identificar y ubicar geográficamente los criaderos presentes en el área. El uso de sistemas de información geográfica con alta resolución para identificación y vigilancia de criaderos es una herramienta útil para la planeación de estrategias de manejo ambiental. La actualización de mapas en tiempo real según el tipo de actividades de control realizadas es conveniente para la vigilancia y la evaluación de la efectividad de las medidas de control adoptadas (20).

Aunque los casos de malaria autóctonos se presentaron en todos los grupos de edad, llama la atención la alta incidencia de malaria en los menores de quince años, similar a lo encontrado en el área urbana de Buenaventura, donde el riesgo de infectarse con malaria fue más alto en los menores, especialmente por debajo de los diez años, y disminuía con la edad (21). Las ocupaciones rurales que implican movilizarse al bosque constituyen un factor de riesgo para malaria en residentes de áreas urbanas y los infectados pueden funcionar como reservorio para el mantenimiento de la transmisión en la ciudad (21). Sin embargo, al igual que en Buenaventura (21), en Quibdo la presencia de casos de malaria en niños, estudiantes y amas de casa sugiere la existencia de transmisión autóctona e intradomiciliaria.

La presencia de casos de malaria en el año anterior a la entrevista en personas infectadas por $P$. vivax se debe a la posible existencia de recaídas. La historia de infecciones previas de malaria en el mismo hogar supone la existencia de agregados de transmisión determinados posiblemente por factores ambientales, económicos, culturales o genéticos (22). La presencia de características genéticas que determinan la susceptibilidad a la infección por malaria puede influir en la epidemiología de la malaria en la ciudad. En Quibdó, la mayoría de casos de malaria por $P$. falciparum se presentaron en personas de raza negra; lo anterior podría explicarse por la ausencia de receptores Duffy necesarios para la infección por $P$. vivax (23). La distribución racial en los diferentes barrios de la ciudad también podría determinar la distribución de las especies de Plasmodium.

El toldillo no impregnado como medida de protección personal se utiliza ampliamente entre la población urbana de Quibdó (> $60 \%$ de la población) y parece ser una estrategia de control efectiva (4). Así mismo, en la región amazónica de Colombia se encontró que el uso del toldillo disminuye el riesgo de infección por malaria en un $50 \%$ comparado con no emplearlo (24). Considerando la alta disponibilidad del toldillo en la población urbana de Quibdó, podría considerarse la implementación de campañas de impregnación de toldillos. Sin embargo, la implementación de esta estrategia en lo posible debe ser validada con estudios entomológicos de hábitos de picadura del vector. El control de 
malaria en las áreas identificadas como zonas de mayor riesgo en la ciudad de Quibdó se podría alcanzar a través de un manejo integrado que incluya identificación y control de criaderos, diagnóstico y tratamiento temprano y adecuado de los pacientes, uso de medidas de protección personal y educación comunitaria en salud (20). Las actividades de manejo ambiental orientadas a la eliminación de criaderos han demostrado ser sostenibles y costo efectivas por la facilidad operacional, el bajo costo y por la participación de la comunidad en las actividades de control (20).

Para estimar la magnitud de problema de malaria urbana en Quibdó y medir el verdadero impacto de las medidas de control implementadas es necesario discriminar sistemáticamente los casos diagnosticados en Quibdó en autóctonos e importados. La definición utilizada en este estudio es práctica, pero puede ser imprecisa debido a que los residentes de fuera de Quibdó que permanecen largo tiempo en la ciudad y allí adquieren la infección serían erróneamente clasificados como importados. En vista de que el riesgo de adquirir malaria en la ciudad se considera menor de acuerdo con las relativamente altas incidencias de malaria que se reportan en el área rural y otros municipios del departamento (8), el error de clasificación de un caso importado en no residentes de Quibdó tendría un bajo impacto en los resultados del estudio y consideramos que esta definición puede usarse rutinariamente en el programa de vigilancia y control de malaria. Así mismo, considerar el barrio de residencia de los casos clasificados como autóctonos como el sitio probable de infección es conveniente, ya que si bien los casos pueden adquirirse en otro barrio, establecer los patrones y cuantificar la movilidad dentro de la ciudad es metodológicamente complicado. Por lo tanto, consideramos que el análisis basado en el lugar de residencia da una estimación aceptable de la distribución del riesgo de malaria dentro de la ciudad, el cual debe correlacionarse con los hallazgos entomológicos.

\section{Agradecimientos}

Agradecemos la colaboración de DASALUDChocó y el Programa de Control de Vectores (ETV), a Ana Luisa Arriaga, Ubertina Córdoba,
Freddy Córdoba y las autoridades de salud de la ciudad de Quibdó. Agradecemos al personal de los hospitales, clínicas y centros de salud de la ciudad de Quibdó por permitirnos realizar este estudio en sus instituciones. Agradecemos a los encuestadores, María Helena Chacón y Rubiela Giraldo, por su contribución, y a los pacientes sin quienes este estudio no hubiese podido realizarse.

\section{Conflicto de intereses}

Ninguno.

\section{Financiación}

Este estudio fue financiado por WHO/TDR (Tropical Diseases Research) contrato (A00859). Lyda Osorio fue apoyada por una beca del Instituto Colombiano para el Desarrollo de la Ciencia y la Tecnología "Francisco José de Caldas", Colciencias, y Johanna Ochoa recibe una beca del Programa de Jóvenes Investigadores de Colciencias.

\section{Referencias}

1. Instituto Nacional de Salud. Informe de enfermedades transmitidas por vectores (ETV), 2004. Inf Quinc Epidemiol Nac 2005;10:33-48.

2. Instituto Nacional de Salud. Situación epidemiológica de las enfermedades transmitidas por vectores 20032004. Boletín Epidemiológico Semanal -SIVIGILASistema de vigilancia en Salud Pública. Semana epidemiológica 8. Febrero 22-28 del 2004.

3. Serra M, Gómez E, Carvajal R, Banguero M, Olano V, Córdoba F. El mundo de la malaria como una estrategia para el control de la malaria a nivel municipal. Santafé de Bogotá D.C: Fundación FES; 2000.

4. Osorio L, Todd J, Bradley DJ. Travel histories as risk factors in the analysis of urban malaria in Colombia. Am J Trop Med Hyg 2004;71:380-6.

5. Subdirección de Metereología-Estación el Caraño. Información climatológica. Santafé de Bogotá D.C.: Instituto de Hidrología, Metereología y Estudios Ambientales (IDEAM); 2000.

6. Jimeno M, Sotomayor M, Valderrama L. Chocó: biodiversidad cultural y medio ambiente. Santafé de Bogotá D.C: Fondo FEN; 1995.

7. DANE. Censo 1993. Chocó - Colombia. 1998: Santafé de Bogotá D.C: DANE; 1998.

8. Arriaga A. Statistics of malaria in Chocó. Chocó: DASALUD; 2000.

9. Kirkwood B. Essentials of medical statistics. London: Blackwell Science Ltd.; 1988. 
10. Gamage-Mendis AC, Carter R, Mendis C, De Zoysa AP, Herath PR, Mendis KN. Clustering of malaria infections within an endemic population: risk of malaria associated with the type of housing construction. Am J Trop Med Hyg 1991;45:77-85.

11. Keiser J, Utzinger J, Caldas de Castro M, Smith TA, Tanner M, Singer BH. Urbanization in sub-saharan Africa and implications for malaria control. Am J Trop Med Hyg 2004;71:118-27.

12. Trape JF, Lefebvre-Zante E, Legros F, Ndiaye G, Bouganali $\mathrm{H}$, Druilhe $\mathrm{P}$, et al. Vector density gradients and the epidemiology of urban malaria in Dakar, Senegal. Am J Trop Med Hyg 1992;47:181-9.

13. Trape JF, Lefebvre-Zante $E$, Legros $F$, Druilhe $P$, Rogier C, Bouganali $\mathrm{H}$, et al. Malaria morbidity among children exposed to low seasonal transmission in Dakar, Senegal, and its implications for malaria control in tropical Africa. Am J Trop Med Hyg 1993;48:748-56.

14. Thompson R, Begtrup K, Cuamba N, Dgedge M, Mendis C, Gamage-Mendis A, et al. The Matola malaria project: a temporal and spatial study of malaria transmission and disease in a suburban area of Maputo, Mozambique. Am J Trop Med Hyg 1997;57:550-9.

15. Staedke SG, Nottingham EW, Cox J, Kamya MR, Rosenthal PJ, Dorsey G. Short report: proximity to mosquito breeding sites as a risk factor for clinical malaria episodes in an urban cohort of Ugandan children. Am J Trop Med Hyg 2003;69:244-6.

16. Carter R, Mendis KN, Roberts D. Spatial targeting of interventions against malaria. Bull World Health Organ 2000;78:1401-11.
17. Méndez F, Carrasquilla G. Epidemiología de la malaria en el área urbana de Buenaventura: análisis de la ocurrencia en el periodo 1987-1993. Colombia Médica 1995;26:77-85.

18. Olano V, Carrasquilla G, Mendez F. Transmission of urban malaria in Buenaventura, Colombia: entomological features. Rev Panam Salud Publica 1997;1:287-94.

19. Brochero HL, Rey G, Buitrago LS, Olano VA. Biting activity and breeding sites of Anopheles species in the municipality of Villavicencio, Meta, Colombia. J Am Mosq Control Assoc 2005;21:182-6.

20. Caldas de Castro M, Yamagata Y, Mtasiwa D, Tanner M, Utzinger J, Keiser J, et al. Integrated urban malaria control: a case study in Dar es Salaam, Tanzania. Am J Trop Med Hyg 2004;71(Suppl. 2):10317.

21. Méndez F, Carrasquilla G, Muñoz A. Risk factors associated with malaria infection in an urban setting. Trans R Soc Trop Med Hyg 2000;94:367-71.

22. Greenwood BM. The microepidemiology of malaria and its importance to malaria control. Trans R Soc Trop Med Hyg 1989;83(Suppl.):25-9.

23. Miller LH, McAuliffe FM, Mason SJ. Erythrocyte receptors for malaria merozoites. Am J Trop Med Hyg 1977;26:204-8.

24. Alexander N, Rodriguez M, Perez L, Caicedo JC, Cruz J, Prieto G, et al. Case-control study of mosquito nets against malaria in the Amazon region of Colombia. Am J Trop Med Hyg 2005;73:140-8. 